\title{
Shielding Effectiveness of Ceramic Bodies Produced with Natural Zeolite
}

\author{
B. KANBEROĞLU ${ }^{a}$ AND A. ŞÜKRAN DEMIRKIRAN ${ }^{b}$ \\ ${ }^{a}$ Sakarya University, Faculty of Engineering, Department of Electrical and Electronics Engineering, Sakarya, Turkey \\ ${ }^{b}$ Sakarya University, Faculty of Engineering, Department of Metallurgical and Materials Engineering \\ Sakarya, Turkey
}

\begin{abstract}
In this study, electromagnetic shielding effectiveness of ceramic bodies produced with natural zeolite illuminated by an electromagnetic pulse is investigated. A matrix model is used to calculate the propagation of electromagnetic pulse through sample. Shielding effectiveness of sample is determined for both transmitted electric and magnetic fields. Mathematical theory of the interaction and the shielding effectiveness of the sample is determined as a function of frequency, thickness of the material and the incidence angle of electromagnetic pulse. Zeolites used in this study were supplied from ETI Holding Company located in Turkey. Water was added as a binder and disc samples were shaped by uniaxial dry pressing at pressing pressure of 1.5 tone. Samples were fired in an electric furnace with a heating rate of $10^{\circ} \mathrm{C} / \mathrm{min}$ at $1150{ }^{\circ} \mathrm{C}$ with a period of $60 \mathrm{~min}$. Electrical measurements are performed to determine the dielectric constant and dielectric loss tangent at $25^{\circ} \mathrm{C}$ constant temperature between frequency range from $1 \mathrm{kHz}$ up to $2 \mathrm{MHz}$.
\end{abstract}

DOI: 10.12693 /APhysPolA.125.642

PACS: 81.05.Je, 07.50.Hp, 13.40.-f, 72.20.-i

\section{Introduction}

With the increasing number of commercial, military and scientific electrical and electronic devices, shielding becomes an important technique for reducing electromagnetic interference and protecting electronic devices and equipments against electromagnetic waves. Shielding theory is based on transmission of electromagnetic wave through material and reflection from the material. Determination of the shielding effectiveness (SE) of multilayered structures, polymers, conducting composites and materials are of interest in many technological applications such as microelectronic devices, microwave absorbers, electromagnetic compatibility and aerospace applications.

The effects of materials as copper, steel, and aluminum on SE [1], SE of conductive polymers polyacetylene and PBT [2], laminated shields constructed with polymers and metals [3] and three-layered Pani/Pu films [4] were evaluated as a function of frequency, thickness, and polarization. A theoretical plane-wave matrix model is used to determine the transmission of electromagnetic fields on the material $[5,6]$.

In this paper, plane wave electromagnetic shielding behavior of ceramic bodies produced with natural zeolite is investigated via measured permittivity and tangent loss measured as a function of thickness, incidence angle, and frequency. Ceramic materials are very good insulators due to their resistance against the penetration of electricity and widely used in the microelectronic devices, ceramic heaters, semiconducting elements as in power transmission lines [7]. The dielectric and electrical properties of the sample measured using HP4980A LCR meter after coating silver layers on the 2 surfaces of the pellet as electrode. A matrix model is applied to compute the reflection and transmission of EMP and SE of sample.

\subsection{Electrical properties of sample}

The complex permittivity of a material can be expressed as a relative value $\varepsilon=\varepsilon_{0} \varepsilon_{\mathrm{r}}=\varepsilon_{0}\left(\varepsilon_{\mathrm{r}}^{\prime}-\mathrm{j} \varepsilon_{\mathrm{r}}^{\prime \prime}\right)$ in frequency domain and determined from the measured parameters [7]. Permittivity describes the interaction of material with an electric field. The real part of permittivity referred as dielectric constant in engineering applications and imaginary part of permittivity affect energy absorption and attenuation. The ratio of imaginary part to real part of permittivity, $\tan \delta=\varepsilon_{\mathrm{r}}^{\prime \prime} / \varepsilon_{\mathrm{r}}^{\prime}$ is called the loss tangent (material dissipation factor) $[8,9]$.

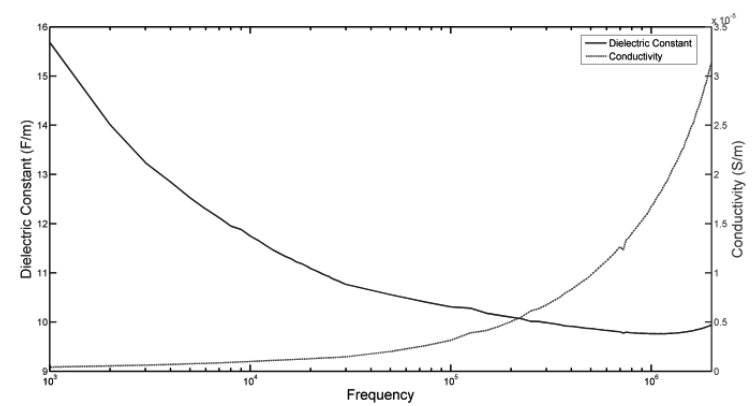

Fig. 1. Dielectric constant $\varepsilon_{\mathrm{r}}^{\prime}$ and conductivity $\sigma$ of the sample at selected frequencies.

The conductivity is determined via imaginary part of permittivity [10]:

$$
\sigma(f)=w \varepsilon_{0} \varepsilon_{\mathrm{r}}^{\prime \prime}(f)
$$

where $\varepsilon_{0}$ is the permittivity of free space and $w$ is the angular frequency. Frequency-dependent dielectric constant $\varepsilon_{\mathrm{r}}^{\prime}$ and conductivity $\sigma$ are also shown in Fig. 1, respectively. 


\subsection{Electromagnetic interaction}

The reflection and transmission of EMP on the sample are presented in Fig. 2. The direction of incident EMP is defined by elevation $(\varphi)$ angle. The sample is defined by constitutive quantities conductivity $\left(\sigma_{\mathrm{s}}\right)$, permittivity $\left(\varepsilon_{\mathrm{s}}\right)$, permeability $\left(\mu_{\mathrm{s}}\right)$ and sample thickness $\left(d_{\mathrm{s}}\right)$.

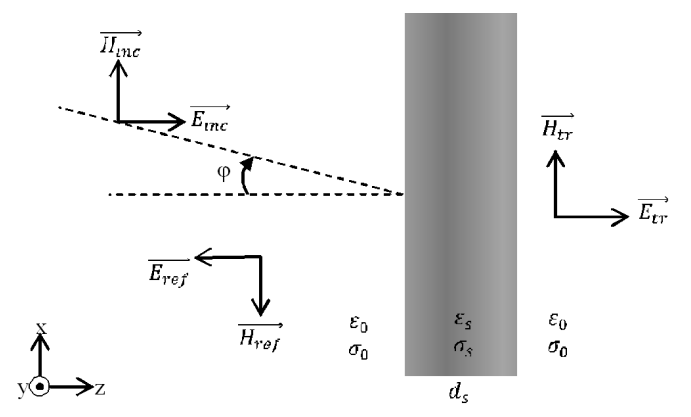

Fig. 2. The reflection and transmission of EMP on sample.

A transfer matrix is used to determine the transmitted and reflected electromagnetic waves on the sample. The matrix model defines the linear connection of electromagnetic field vectors at the sample boundaries $(z=0$ and $\left.z=d_{\mathrm{s}}\right)$ :

$$
\left[\begin{array}{l}
E_{z} \\
E_{y} \\
H_{z} \\
H_{y}
\end{array}\right]_{\mathrm{tr}}=[T]\left[\begin{array}{l}
E_{z} \\
E_{y} \\
H_{z} \\
H_{y}
\end{array}\right]_{\mathrm{inc}} .
$$

Transfer matrix is a four by four matrix due to variation of incidence angles. For $\varphi=0$ incidence angle, transfer matrix can be reduced to $2 \times 2$ matrix

$$
\begin{aligned}
& T= \\
& {\left[\begin{array}{cccc}
\cos \left(k_{z} d\right) & 0 & 0 & \frac{Z k_{z}}{\mathrm{i} k} \sin \left(k_{z} d\right) \\
0 & \cos \left(k_{z} d\right) & \frac{Z k}{\mathrm{i} k_{z}} \sin \left(k_{z} d\right) & 0 \\
0 & \frac{\mathrm{i} k_{z}}{Z k} \sin \left(k_{z} d\right) & \cos \left(k_{z} d\right) & 0 \\
\frac{k}{\mathrm{i} Z k_{z}} \sin \left(k_{z} d\right) & 0 & 0 & \cos \left(k_{z} d\right)
\end{array}\right] .}
\end{aligned}
$$

Wave number and wave impedance of the sample and complex frequency $(s=\Omega+\mathrm{j} w)$ are used in transfer matrix to determine the interaction of EMP and given below

$$
\begin{aligned}
& Z=\sqrt{\frac{s \mu_{\mathrm{s}}}{\sigma_{\mathrm{s}}+s \varepsilon_{\mathrm{s}}}}, \\
& k=\sqrt{-s \mu_{\mathrm{s}}\left(\sigma_{\mathrm{s}}+s \varepsilon_{\mathrm{s}}\right)} .
\end{aligned}
$$

The electromagnetic SE is specified as the reduction of electromagnetic field strength caused by material. SE depends on a number of parameters, most notable of which are conductivity, permittivity, permeability, thickness of the material, incidence angle, and polarization [4-6]. The shielding effectiveness is defined as the ratio of the transmitted field strength $\left(E_{\mathrm{tr}}, H_{\mathrm{tr}}\right)$ to incident field strength $\left(E_{\text {inc }}, H_{\text {inc }}\right)$ :

$$
\begin{aligned}
& \mathrm{SE}_{E}=-20 \log \left(\frac{E_{\mathrm{tr}}}{E_{\mathrm{inc}}}\right), \\
& \mathrm{SE}_{H}=-20 \log \left(\frac{H_{\mathrm{tr}}}{H_{\mathrm{inc}}}\right) .
\end{aligned}
$$

A plane electromagnetic pulse (EMP) described by a double exponential waveform is applied on the sample

$$
E(t)=E_{0}\left(\mathrm{e}^{-\alpha t}-\mathrm{e}^{-\beta t}\right) .
$$

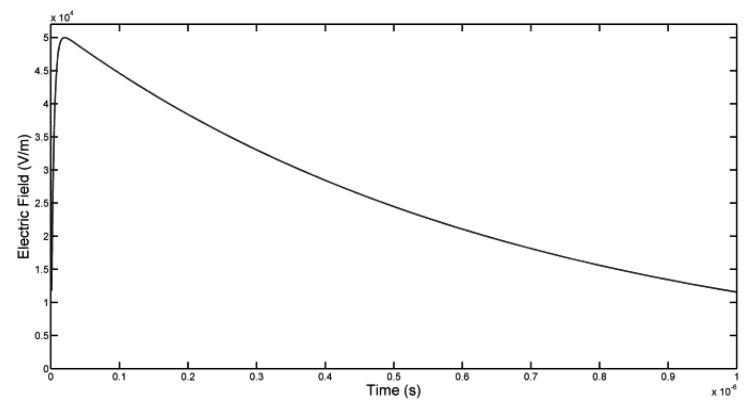

Fig. 3. Incident EMP-wave in time domain.

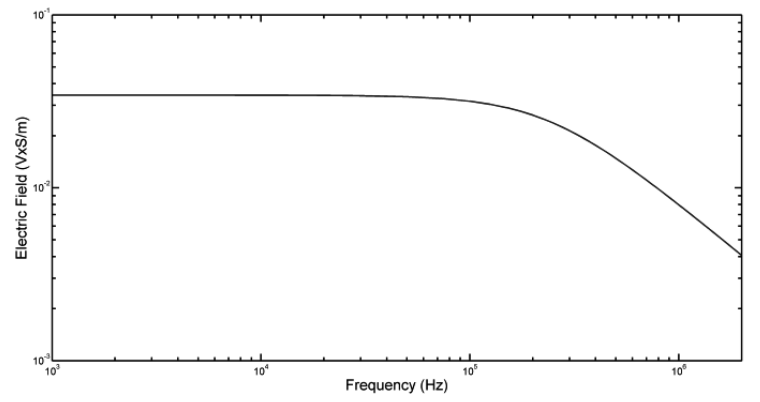

Fig. 4. Incident EMP-wave in frequency domain.

Mathematical theory of the interaction is realized in frequency domain. Therefore, the Laplace transform is used to transfer the time dependent waveform equation into frequency domain. EMP parameters are $\alpha=$ $1.5 \times 10^{6} \mathrm{~s}^{-1}, \beta=2.6 \times 10^{8} \mathrm{~s}^{-1}$, and $E_{0}=50 \times 10^{3} \mathrm{~V} / \mathrm{m}$. The EMP in time and frequency domain is shown in Figs. 3 and 4.

\section{Results}

In this paper, ceramic bodies produced with natural zeolite is considered as a single shield with a thickness of $5 \mathrm{~mm}$. EMP is applied to the boundary of the sample with an incidence angle $\varphi=0$.

The dispersive effect of the sample on EMP and electric and magnetic field at observation point are calculated using Eq. (2) for $1 \mathrm{kHz}-2 \mathrm{MHz}$ frequency range. The electromagnetic SE is evaluated using Eq. (5) and Eq. (6) via obtained electric and magnetic fields. Electrical and magnetic SE of the sample are shown in Figs. 5 and 6 , respectively.

According to Fig. 5, sample presents an electromagnetic SE about $9 \mathrm{~dB}$. Electrical $\mathrm{SE}$ values vary from 


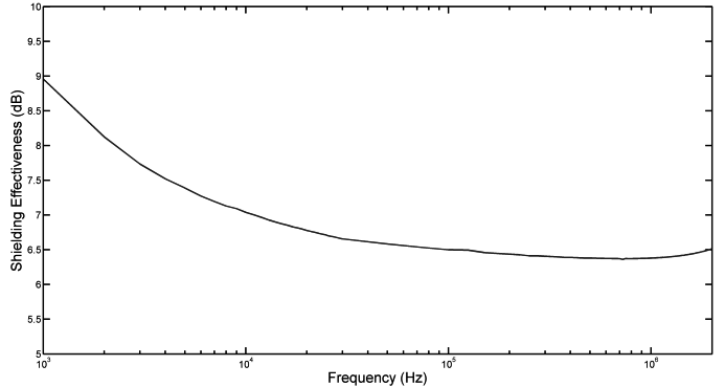

Fig. 5. Electrical SE of the sample.

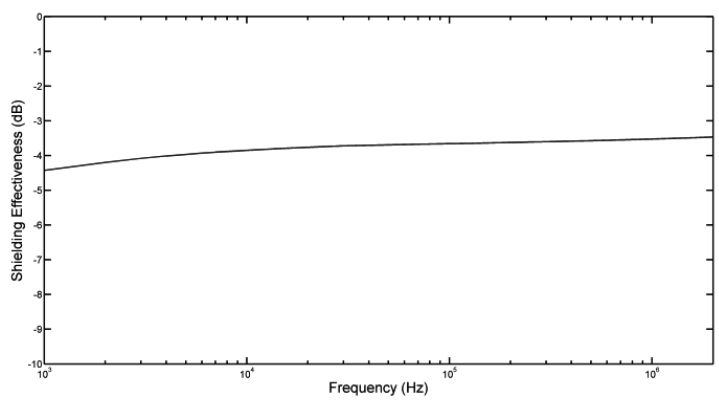

Fig. 6. Magnetic SE of the sample.

$6.5 \mathrm{~dB}$ to $9 \mathrm{~dB}$ and from $1 \mathrm{kHz}$ to $2 \mathrm{MHz}$. Electrical SE of low frequency is higher than that of high frequency. At lower frequencies, SE decreases clearly from $9 \mathrm{~dB}$ to $7 \mathrm{~dB}$ levels but at higher frequencies SE changes slightly with increasing frequency.

Magnetic SE of the sample is shown in Fig. 6. Opposite to electrical SE results, magnetic SE of high frequency is better than low frequency and it is clear that magnetic $\mathrm{SE}$ increases with frequency. Magnetic SE values of the sample range from $-3.46 \mathrm{~dB}$ to $-4.42 \mathrm{~dB}$ and from $1 \mathrm{kHz}$ to $2 \mathrm{MHz}$.

To achieve higher SE, a scenario of increasing conductivity is considered with 3 different conductivity values higher than sample conductivity for constant permittivity and permeability. Results are shown in Figs. 7 and 8 . It is obvious that electrical SE improves with increasing conductivity. The increase of the conductivity improves

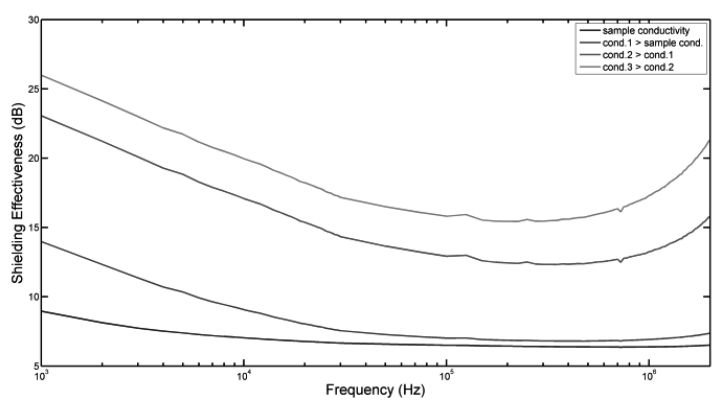

Fig. 7. Electrical SE with enhanced conductivity. the electrical SE as shown in Fig. 7 but does not affect the magnetic SE as well as electrical SE. Magnetic SE results change slightly with conductivity as shown in Fig. 8.

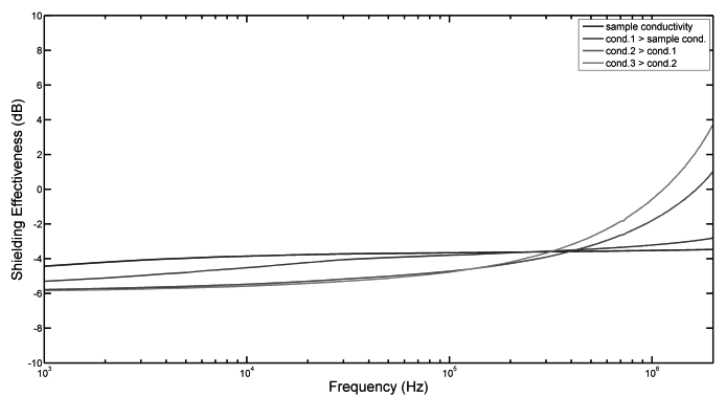

Fig. 8. Magnetic SE with enhanced conductivity.

\section{Conclusions}

In this study, the shielding performance of ceramic bodies produced with natural zeolite is considered via a plane wave approach. A transfer matrix is used to calculate the SE of the sample. Up to $9 \mathrm{~dB}$ attenuation is achieved at frequency range from $1 \mathrm{kHz}$ to $2 \mathrm{MHz}$. An increasing conductivity consideration of the sample shows the possibility of achieving higher electrical SE with improving sample.

\section{Acknowledgments}

Thanks to ETI Holding Company in Turkey for their support for zeolites used in this study.

\section{References}

[1] H. Saadi, R. Oussaid, in: IEEE Int. Conf. on Signal Processing and Communications (ICSPC 200\%), Dubai, Ed.: M.E. al-Mualla, IEEE Region 8, Dubai 2007, p. 999.

[2] K. Naishadham, IEEE Trans. Electromagn. Compat. 34, 47 (1992)

[3] P.V.Y. Jayasree, V.S.S.N.S. Baba, B.P. Rao, in: Electromagnetic Interference and Compatibility (INCEMIC), Bangalore (India), Ed.: D.C. Pande, IEEE, Bangalore 2008, p. 463.

[4] H.N. Nhan, J.L. Miane, J.L. Wojkiewicz, in: 1st Int. Conf. on Communications and Electronics (ICCE), Hanoi, Eds.: N.Q. Trung, K. Tanaka, H. Lee, N.H. Than, IEEE, Hanoi 2006, p. 482.

[5] M. Born, E. Wolf, Principles of Optics, Cambridge University Press, UK 1999, p. 56.

[6] W. Blumer, N. Ari, IEE Proc.-A 41, 199 (1991).

[7] A.S. Demirkiran, R. Artir, E. Avci, Ceram. Int. 36, 917 (2010).

[8] V. Komarov, S. Wang, J. Tang, in: The Wiley Encyclopedia of RF and Microwave Engineering, Ed. K. Chang, Wiley, New York 2005, p. 3693.

[9] Agilent Solutions for Measuring Permittivity and Permeability with LCR Meters and Impedance Analyzers, Application Note 1369-1 (2008).

[10] C.A. Balanis, Advanced Engineering Electromagnetics, Wiley, USA 1989, p. 42. 